Revista de Psicología Vol. 39 (2), 2021 (e-ISSN 2223-3733)

\title{
Percepción de eficacia en el control de la corrupción y su relación con el clima socioemocional y la identidad nacional en el Perú: Un estudio experimental
}

\author{
Hernán Chaparro ${ }^{1}$, Agustín Espinosa ${ }^{2} \&$ Darío Páez $^{3}$ \\ Universidad de Lima-Perú, Pontificia Universidad Católica del Perú-Perú, \\ Universidad de País Vasco-España
}

Este artículo revisa la definición de corrupción y sus correlatos individuales, macro y micro sociales. En un estudio de campo experimental con una muestra representativa peruana $(N=$ $1,254)$, se informó a los participantes de una gestión jurídica del control de la corrupción de alta eficacia (un $95 \%$ de los casos fueron juzgados y sancionados) versus un control de baja eficacia (solo un $5 \%$ fue juzgado y sancionado). La información inducida sobre la eficacia en el control y sanción de la corrupción provocó una mayor percepción de eficacia colectiva para tratar la desviación y una mejor balanza de clima socioemocional en el grupo de alta eficacia. La inducción experimental de mayor eficacia interactuó con el interés en la política y produjo mayor percepción de eficacia en el control de corrupción y mejor balanza de clima socioemocional en las personas de alto interés. Adicionalmente, se observa que la identificación con el Perú aumentó solo en las personas que confiaban previamente en el sistema de justicia (poder judicial) y que fueron informadas de una alta eficacia de control de la corrupción. Se discuten los resultados en el marco de la dinámica grupal de control de la desviación e identidad grupal y la importancia del contexto institucional y del nivel de interés en la política.

Palabras clave: Corrupción, Clima Socioemocional, Confianza Institucional, Interés en la Política.

Perception of efficacy in the control of corruption and its relation with socioemotional climate and Peruvian national identity: An experimental study

This article reviews the definition of corruption and its individual, macro and micro-social correlates. In an experimental field study with a representative Peruvian sample $(\mathrm{N}=1254)$, participants were informed of a highly effective legal management of corruption control (95\% of cases were tried and punished) versus a control of low efficacy (only 5\% were tried

1 PhD en Psicología. Docente en la Universidad de Lima, Perú. Contacto: hchaparr@ulima. edu.pe. https://orcid.org/0000-0001-5538-074X

$2 \mathrm{PhD}$ en Psicología. Profesor Principal en la Pontificia Universidad Católica del Perú, Perú. Contacto: agustin.espinosa@pucp.pe. https://orcid.org/0000-0002-2275-5792

$3 \mathrm{PhD}$ en Psicología. Catedrático en la Universidad de País Vasco, España. Contacto: dario. paez@ehu.es. https://orcid.org/0000-0002-8459-6037 
and punished). The induced information on the effectiveness in the control and sanction of corruption led to a greater perception of collective efficacy in dealing with deviation and a better balance of socio-emotional climate in the high-efficacy group. The most effective experimental induction interacted with interest in politics and produced a greater perception of efficacy in the control of corruption and a better balance of socio-emotional climate in people of high interest. Additionally, it is observed that the identification with Peru increased only in people who previously trusted the justice system (judicial power) and who were informed of a high efficiency of corruption control. The results are discussed within the framework of the group dynamics of control of deviance and group identity and the importance of the institutional context and the level of interest in politics.

Keywords: Corruption Socio-emotional Climate, Institutional Trust, Interest in Politics

\section{Percepçáo de eficácia no controle da corrupçáo e sua relaçáo com o clima socioemo-} cional e a identidade nacional peruana: um estudo experimental.

Este artigo analisa a definição de corrupção e os seus correlatos individuais, macro e microssociais. Num estudo de campo experimental, com uma amostra peruana representativa ( $\mathrm{N}$ = 1254), os participantes foram informados que existia uma gestáo legal eficaz no controlo da corrupçáo ( $95 \%$ dos casos foram julgados e punidos) versus ineficaz (apenas 5\% foram julgados e punidos). A informação sobre a eficácia no controlo e punição da corrupção induziu maior percepção de eficácia coletiva no controlo do desvio e melhor clima emocional. A indução experimental mais efetiva interagiu com o interesse na política e produziu uma maior percepção de eficácia no controlo da corrupção e melhor clima na condição de eficácia elevada. Além disso, observa-se que a identificação com o Peru aumentou apenas em pessoas que confiavam previamente na justiça e que foram informadas sobre eficácia elevada no controlo de desvio. Os resultados são discutidos no âmbito da dinâmica de grupo de controle de desvio e identidade de grupo e a importância do contexto institucional e o nível de interesse em política.

Palavras chave: Corrupção, Clima Socio-emocional, Confiança em Instituições, Interesse em Política.

\section{Perception de l'efficacité dans le contrôle de la corruption et sa relation avec le climat socio-émotionnel et l'identité nationale péruvienne: une étude expérimentale.}

Cet article passe en revue la définition de la corruption et ses corrélats individuels, macro et microsociaux. Dans une étude de terrain expérimentale avec un échantillon péruvien représentatif $(\mathrm{N}=1253)$, les participants ont été informés de la gestion juridique efficace $(95 \%$ des cas ont été jugés) par rapport à inefficace (seulement $5 \%$ ont été jugés). La perception de l'efficacité du groupe induit a provoqué une plus grande perception de l'efficacité collective pour traiter la déviation et un meilleur climat émotionnel. L'induction expérimentale plus efficace a interagi avec l'intérêt pour la politique et a produit une plus grande perception de l'efficacité dans le contrôle de la corruption et un meilleur climat chez les personnes de grand intérêt. De plus, il est observé que l'identification avec le Pérou n'a augmenté que chez les personnes qui avaient auparavant confiance en la justice et qui étaient informées de l'efficacité élevée du contrôle des écarts. Les résultats sont discutés dans le cadre de la dynamique de groupe du contrôle de la déviation et de l'identité du groupe et de l'importance du contexte institutionnel et du niveau d'intérêt pour la politique.

Mots clés : corruption, climat socio-émotionnel, confiance dans les institutions, intérêt pour la politique. 
La corrupción se define como el conjunto de actos en que se produce un abuso de la función pública para beneficios privados (Transparency International, 2018). También puede ser definida como el abuso de un poder delegado para obtener beneficios privados, que sesga decisiones administrativas y políticas, desvía recursos y socava el desarrollo sostenible de una sociedad (Boehm, Isaza, \& Villalba, 2015). La corrupción comprende acciones no éticas, anti-normativas o deshonestas, que en particular desvían bienes públicos a fines privados o a fines distintos de los objetivos fijados desde la función pública (Belle \& Cantarelli, 2017). Aunque su expresión conductual es variada, las manifestaciones más frecuentes de la corrupción consisten en la recepción o el pago de sobornos para acceder a beneficios que a su vez degradan, los servicios y bienes públicos que deberían estar disponibles a todos los ciudadanos (Transparencia Internacional, 2019). Entre las condiciones políticas y sociales que se plantean como causas de la corrupción se encuentran las siguientes:

(a) Una débil separación de las esferas pública y privada, y la apropiación generalizada de los recursos públicos por parte de determinados grupos de poder. Sin embargo, la eficacia de las instituciones y la apertura gubernamental se asocian negativamente a la corrupción (Judge, McNatt, \& Xu, 2011);

(b) Relaciones verticales y clientelistas, basadas en criterios identitarios de estatus y poder (p.e. parentesco, etnia, religión) que priman sobre las relaciones horizontales y basadas en derechos ciudadanos igualitarios. La primacía de la confianza particularista o vinculada a un grupo social y un bajo capital social son predictores de corrupción en una sociedad (Heath, Richards, \& de Graaf, 2016).

(c) Períodos de transición política en una sociedad y los regímenes híbridos o autoritarios, a menudo, están más asociados a la corrupción que los momentos de estabilidad política y la presencia de 
regímenes democráticos consolidados (UK International Development Department, 2015).

Donde se encuentra presente, la corrupción tiene un impacto económico negativo a modo de círculo vicioso. Es decir, a más corrupción, menor crecimiento económico lo que, a su vez, lleva a más corrupción. Sin embargo, la relación entre desarrollo económico y corrupción no es simple y lineal; pues, aunque suele encontrarse con mayor frecuencia en países poco desarrollados, pero con grandes riquezas naturales; existen países de bajo desarrollo económico con bajos índices de corrupción (p.e. Uruguay) y países de alto desarrollo económico con altos niveles de corrupción (p.e. Italia) (Judge et al., 2011).

La corrupción tiene un impacto desproporcionado en los grupos más vulnerables, pues tiende a aumentar la desigualdad, a partir de la reducción o mala asignación del gasto social (UK International Development Department, 2015). Estudios recientes muestran que las percepciones de corrupción y el conflicto social se asocian de manera ambivalente, pues por una parte motivan procesos de cambio social, pero por otra, al debilitar el sistema político e institucional sirve para mantener regímenes débiles o autoritarios (Espinosa, Pacheco, Acosta et al., 2020).

La corrupción permite a ciertas élites legitimarse a expensas del debilitamiento del Estado. Esto porque la legitimidad política del gobierno se debilita, si este permite a algunos obtener ganancias privadas desproporcionadas a expensas de otros (Rose-Ackerman, 2001). En ese sentido, existe amplio consenso respecto al impacto negativo de la corrupción sobre la consolidación y el desempeño de las democracias (Morales, 2009). Algunos estudios sugieren que la relación entre corrupción y democracia es de causalidad. Es decir, que mayores niveles de democratización en una sociedad reducen las percepciones de corrupción al interior de la misma (Jaramillo, 2011). Asimismo, se ha planteado inclusive un vínculo entre corrupción y nivel democrático de una nación. Por ejemplo, en una escala de evaluación de la corrupción de uno a cien, en la que puntuaciones altas equivalen a menor corrupción se encontró que la puntuación media de las socie- 
dades categorizadas como democracias plenas era de 75 puntos, de 49 puntos en las "democracias imperfectas», 35 puntos en los «regímenes híbridos» y 30 puntos en los regímenes «autocráticos» (Transparencia Internacional, 2018).

Un meta-análisis de 137 estudios examinó los factores situacionales o microsociales y psicológicos que reforzaban la conducta de hacer trampa, aprovecharse deshonestamente de recursos y otros indicadores similares. Las variables dependientes eran mediciones sobre falta de ética como el comportamiento real, la intención auto-reportada de comportarse deshonestamente y el juicio auto-informado de conductas contrarias a la ética. Los resultados encontraron que el factor socio-ambiental que más reforzaba las medidas de falta de ética era la justificación de la conducta corrupta a partir de: (1) su minimización (p.e. "no es tan grave"), (2) el reencuadre positivo (p.e. "es solo una forma de restaurar una situación injusta”), (3) el devaluar a las víctimas (p.e "es culpa de ellos que les robemos") (Belle \& Cantarelli, 2017). Consistentemente, con base en 13 estudios, se encontró que cuanto más el entorno les permita a las personas racionalizar su comportamiento desviado, es más probable que estas se comporten de manera poco ética ( $r$ media=.44, Bozerman \& Gino, 2012).

Con base en 4 estudios se encontró que otro factor que refuerza la corrupción, es la presión del tiempo o fijarse metas muy altas que deban cumplirse en poco tiempo ( $r$ media=.32). Por otra parte, el predominio de una imagen de sí mismo egocéntrica, independiente y poco relacional se asoció en 20 estudios con un tamańo del efecto de $r$ media=.27 a la corrupción. Otro factor que favorece la corrupción es la exposición a la deshonestidad incremental, consistente en la escalada desde pequeñas transgresiones a convenciones sociales a actos corruptos más graves paulatinamente. Siete estudios demuestran que las situaciones donde están presentes la riqueza y la desigualdad desencadenan en las personas envidia y avaricia, por lo que mediante actos corruptos quieren reparar la situación injusta ( $r$ media=.26). 26 estudios muestran que el ser testigo o ver a pares cometer actos deshonestos incide en las prácticas corruptas como una forma de influencia 
social ( $r$ media=.25). 33 estudios muestran que enmarcar la evitación de una conducta corrupta como una pérdida (si no se comete el acto, el corrupto perderá un monto de dinero) incrementa la adhesión a la corrupción ( $r$ media=.24); asimismo, cinco estudios muestran que cuando una situación corrupta se describe como una ganancia, también se incrementa la adhesión a la corrupción ( $r$ media=.25) (Philip \& David-Barrett, 2015).

Por otra parte, los factores que refuerzan la conducta normativa e inhiben la corrupción son; en primer lugar, el monitoreo o control de la conducta de los empleados; así, 19 estudios muestran que donde el control destaca y pone de relieve la existencia de actos corruptos, al mismo tiempo que señala que quienes cometan estos actos serán sancionados, inhibe la adhesión a la corrupción ( $r$ media=-.42). 12 estudios muestran que la disposición a mantener una imagen positiva de sí mismas disminuye las conductas poco éticas como la corrupción ( $r$ media=-.28). Finalmente, 15 estudios muestran que recordatorios morales que hacen que las personas tengan presentes códigos éticos inhiben la corrupción ( $r$ media $=-.21$ ) (Belle \& Cantarelli, 2017).

En la tabla 1 se sintetiza bajo la forma del Efecto Binomial Desplegado (Rosenthal, 2005) la importancia de cada factor en el riesgo o protección ante la corrupción, y las acciones de prevención tomadas desde las políticas públicas. De manera específica, el Efecto Binomial Desplegado transforma la correlación en el porcentaje de conductas por encima de la media cuando está presente un factor de corrupción, o el porcentaje de conductas por debajo de la media cuando es un factor de protección. Presuponiendo que la variable conducta corrupta se distribuya de forma normal, se puede pensar que un nivel alto del factor facilitador por encima de la media implica la probabilidad .50 $+r / 2$ de corrupción por encima de la media. A modo de ejemplo, si el factor plantear objetivos desafiantes se asocia $r$ media $=.10$ a la conducta corrupta; esto quiere decir que el $55 \%(.50+.10 / 2=.55)$ de las personas en las que se induce objetivos desafiantes mostrará una respuesta corrupta por encima de la media, comparado con el 45\% $(.50-.10 / 2=.45)$ de personas que no están expuestas a objetivos desa- 
fiantes (Rosenthal \& Rubin, 1982). En este caso, si una institución tiene objetivos elevados, un $55 \%$ de sus miembros se comportará de forma corrupta, por encima de la media, frente a un $45 \%$ de las instituciones que no tienen objetivos elevados.

\section{Tabla 1}

Efecto Binomial Desplegado de Factores de Riesgo/Protección y la acción preventiva en politicas públicas

\begin{tabular}{|c|c|c|c|}
\hline Factor & $\begin{array}{l}\text { Rol del Factor } \\
\text { (Riesgo versus } \\
\text { Protección) }\end{array}$ & $\begin{array}{l}\text { Efecto Binomial } \\
\text { Desplegado }\end{array}$ & $\begin{array}{l}\text { Acción Preventiva } \\
\text { en Política Pública }\end{array}$ \\
\hline $\begin{array}{l}\text { Justificaciones de } \\
\text { la corrupción }\end{array}$ & Riesgo & $\begin{array}{l}72 \% \text { conducta } \\
\text { antinormativa frente } \\
\text { a } 28 \% \text { conducta } \\
\text { normativa }\end{array}$ & $\begin{array}{l}\text { Campañas de } \\
\text { estigmatización y } \\
\text { deslegitimación de } \\
\text { la corrupción }\end{array}$ \\
\hline $\begin{array}{l}\text { Monitoreo o } \\
\text { control de la } \\
\text { conducta ética y } \\
\text { castigo efectivo a } \\
\text { los corruptos }\end{array}$ & Protección & $\begin{array}{l}29 \% \text { conducta } \\
\text { antinormativa frente } \\
\text { a } 71 \% \text { conducta } \\
\text { normativa }\end{array}$ & $\begin{array}{l}\text { Políticas de } \\
\text { transparencia } \\
\text { y control a } \\
\text { proveedores de } \\
\text { bienes públicos } \\
\text { Juicios eficientes } \\
\text { a actividades } \\
\text { corruptas }\end{array}$ \\
\hline $\begin{array}{l}\text { Presión por } \\
\text { hacer muchas } \\
\text { cosas en poco } \\
\text { tiempo }\end{array}$ & Riesgo & $\begin{array}{l}68 \% \text { conducta } \\
\text { antinormativa frente } \\
\text { a } 32 \% \text { conducta } \\
\text { normativa }\end{array}$ & $\begin{array}{l}\text { Proponer plazos } \\
\text { y objetivos de } \\
\text { trabajo realistas } \\
\text { en su viabilidad y } \\
\text { cumplimiento }\end{array}$ \\
\hline $\begin{array}{l}\text { Cultura } \\
\text { individualista } \\
\text { egocéntrica } \\
\text { enfatiza yo } \\
\text { autónomo }\end{array}$ & Riesgo & $\begin{array}{l}63.5 \% \text { conducta } \\
\text { antinormativa frente } \\
\text { a } 36.5 \% \text { conducta } \\
\text { normativa }\end{array}$ & $\begin{array}{l}\text { Reforzar cultura } \\
\text { colectivista } \\
\text { institucional }\end{array}$ \\
\hline
\end{tabular}




\begin{tabular}{|c|c|c|c|}
\hline Factor & $\begin{array}{c}\text { Rol del Factor } \\
\text { (Riesgo versus } \\
\text { Protección) }\end{array}$ & $\begin{array}{l}\text { Efecto Binomial } \\
\text { Desplegado }\end{array}$ & $\begin{array}{l}\text { Acción Preventiva } \\
\text { en Política Pública }\end{array}$ \\
\hline $\begin{array}{l}\text { Existencia } \\
\text { de conductas } \\
\text { corruptas que } \\
\text { aumentan } \\
\text { gradualmente de } \\
\text { menor a mayor } \\
\text { gravedad }\end{array}$ & Riesgo & $\begin{array}{l}62 \% \text { conducta } \\
\text { antinormativa frente } \\
\text { a } 38 \%\end{array}$ & $\begin{array}{l}\text { Política de } \\
\text { tolerancia cero } \\
\text { ante la "pequeña" } \\
\text { corrupción }\end{array}$ \\
\hline $\begin{array}{l}\text { Motivación a } \\
\text { mantener una } \\
\text { buena imagen de } \\
\text { sí mismo }\end{array}$ & Protección & $\begin{array}{l}36 \% \text { conducta } \\
\text { antinormativa frente } \\
64 \% \text { conducta } \\
\text { normativa }\end{array}$ & $\begin{array}{l}\text { Promoción } \\
\text { del énfasis en } \\
\text { mantener una } \\
\text { buena imagen de } \\
\text { sí mismo }\end{array}$ \\
\hline $\begin{array}{l}\text { Exposición a } \\
\text { Recordatorios } \\
\text { morales que } \\
\text { llamen la } \\
\text { atención sobre } \\
\text { las conductas } \\
\text { deshonestas }\end{array}$ & Protección & $\begin{array}{l}39.5 \% \text { conducta } \\
\text { antinormativas } \\
\text { frente } 60.5 \% \\
\text { conducta normativa }\end{array}$ & $\begin{array}{l}\text { Definición de } \\
\text { códigos sobre } \\
\text { conducta ética } \\
\text { y desarrollo de } \\
\text { comunicación } \\
\text { que llame la } \\
\text { atención sobre } \\
\text { consecuencias } \\
\text { de conductas } \\
\text { deshonestas. }\end{array}$ \\
\hline
\end{tabular}

Como se puede constatar el monitoreo o control y castigo a las conductas antinormativas es uno de los procedimientos más eficaces para la inhibición de las mismas. Además de su impacto instrumental, se puede esperar que este control actúe no solo sobre los desviantes y las personas implicadas, sino que, como todo ritual simbólico colectivo, actúe positivamente sobre la cohesión social (cf. Durkheim, 1895/1982). 


\section{Control de la corrupción, clima socioemocional e identidad nacional}

Diversos estudios han corroborado los efectos del control social exitoso de la corrupción en la moral y cohesión en una sociedad. Con base en la Teoría de la Dinámica Subjetiva de los Grupos (Marques, Páez, \& Abrams, 1998), un conjunto reciente de estudios examinó los efectos de la capacidad de un grupo para controlar efectivamente a sus miembros corruptos en la identificación con el grupo nacional (Pinto, Marques, \& Páez, 2016; Hatibovic, Pinto, \& Páez, 2020). La conciencia de la corrupción como una instancia de desviación del grupo podría aumentar la identificación social, cuando el grupo reacciona con fuertes emociones negativas, moviliza el mecanismo de control social y castiga a los desviados. En estas condiciones, la desviación ayuda a mejorar la cohesión social, ya que permite movilizar comportamientos colectivos emocionales y punitivos frente a los miembros desviantes del grupo. Esta excitación emocional y derogación de los desviantes aumenta a su vez, la identificación social. Según Durkheim (1895/1982), la existencia de castigos a los delincuentes, son actividades rituales que fomentan la pertenencia a una comunidad moral. En línea con estas ideas, se realizaron cuatro estudios en los que se informó a los participantes que el grupo nacional se ocupó de los sucesos desviantes de una manera efectiva (versus ineficaz). La efectividad inducida del grupo aumentó la identificación de los participantes con su grupo nacional, reforzó su confianza en el sistema de control social del grupo y evocó un clima emocional más positivo -tanto en Portugal, cuanto en Chile. Por el contrario, la ineficacia grupal inducida generó un clima negativo y debilitó la identificación de los participantes con el grupo nacional en Portugal, aunque no en Chile (Pinto et al., 2016; Hatibovic et al., 2020).

En base a lo descrito, este estudio busca examinar cómo actúan los procesos de control (presentados como eficaces versus ineficaces) de la corrupción en el caso peruano. En este escenario, es esperable que una política pública efectiva de control y sanción de la corrupción mejore la visión que se tiene de la sociedad, específicamente en lo referente al clima emocional y la identidad nacional - aumentando 
la cohesión del grupo nacional. Sobre el particular, estudios previos sugieren que las personas de mayor nivel educativo y más informadas políticamente están más atentas y reaccionan más fuertemente a las deficiencias institucionales y a la corrupción en particular (Charron \& Rothstein, 2016; Anduiza, Gallego, \& Muñoz, 2013; Caillier, 2010). en ese sentido, en contextos de alta corrupción esto las puede llevar a tener una visión tan negativa de la realidad como la de las personas poco interesadas e informadas (Agerberg, 2019). Sin embargo, en contextos de alta corrupción, donde esta es problematizada y donde se perciben mecanismos de control y sanción eficientes hacia la misma, serán las personas más educadas e informadas políticamente quienes mejoren su percepción de la sociedad (Espinosa et al., 2020, Charron \& Rothstein, 2016; Anduiza et al., 2013; Caillier, 2010). Cabe resaltar que el efecto positivo de las acciones anticorrupción eficaces no actúa en el vacío; así, diversos estudios sugieren que instituciones legitimadas por la ciudadanía son un factor contextual que refuerza el efecto de las acciones de control social (Gächter \& Schulz, 2016; Pinto et al., 2016; Hatibovic et al., 2020) o de justicia restaurativa, observándose a modo de ejemplo que el impacto positivo de las Comisiones de la Verdad en América Latina era mayor en aquellos países en los que la confianza en las instituciones democráticas era también mayor (Mathias, Páez, Espinosa et al., 2020).

\section{El estudio en contexto: la corrupción en el Perú actual}

La corrupción ha sido un grave problema a lo largo de la historia del Perú (Quiroz, 2013). Sin embargo, es a partir del año 2000 que la conciencia ciudadana sobre este fenómeno se ha incrementado considerablemente hasta llegar a ser vista, junto con la inseguridad ciudadana, como uno de los problemas más importantes del país (Proética, 2019). Este cambio en las percepciones de la población está vinculado a una serie de denuncias, con alta cobertura en medios, que han implicado a diversos funcionarios de gobiernos locales y regionales pero que además 
han terminado involucrando a políticos, empresarios, e inclusive, a cinco de los últimos presidentes (casi todos los que gobernaron desde 1990 hasta el 2016) en investigaciones, juicios o condenas vinculados a diversos hechos de corrupción.

En este escenario, el sistema judicial, también es evaluado de forma negativa por la ciudadanía (Apoyo Opinión y Mercado, 2006; Instituto de Estudios Peruanos, 2019a). Sin embargo, esto coexiste con una evaluación positiva hacia la Fiscalía por la investigación y acusación de personajes poderosos y sus supuestas redes de corrupción (Instituto de Estudios Peruanos 2019b). No obstante, la evaluación negativa de las instituciones vinculadas a la justicia procedimental ha sido la tendencia. Esta negatividad ha estado relacionada con una baja percepción de eficacia y falta de legitimidad de las mismas, así como por el hecho de que el sistema judicial ha sufrido la injerencia de partidos políticos que en algún momento fueron gobierno, lo que refuerza una imagen de connivencia e impunidad sobre algunas acciones cuestionables de los mismos (La República, 2018; Instituto de Estudios Peruanos, 2018).

En el 2018, las graves denuncias de la existencia de una red de corrupción dentro del Poder Judicial y la Fiscalía llevaron a que se convocara un Referendo donde un $86.56 \%$ de la ciudadanía voto a favor de que se instale una junta para llevar adelante una reforma total del sistema de justicia en el país (Oficina Nacional de Procesos Electorales, 2018). En este escenario político cabe preguntarse ¿Cómo ha procesado la ciudadanía estos sucesos? Y es que en general, la mala imagen del Poder Judicial está vinculada a la evaluación negativa del conjunto del sistema político, (Instituto de Estudios Peruanos, 2019a). Esta percepción de corrupción sistémica a través del tiempo está vinculada a diferentes tipos de actitudes hacia el sistema político en general y dentro de él hacia el sistema judicial. Al respecto, Chaparro (2018) encontró una correlación significativa entre la falta de interés en la política en general y la percepción de baja eficacia política en los ciudadanos. Más aún, se identificaron diverso tipo de segmentos de cultura política donde destacan dos: los llamados Conformistas Desinformados ( $41 \%$ de la población limeña de 18 a más años, $49 \%$ a nivel 
nacional) y los Renegados Insatisfechos (30\% de la población de 18 a más ańos en Lima y 21\% a nivel nacional). En ambos casos, la percepción negativa del funcionamiento del sistema político, entre otros, resultaba en bajos niveles de interés e información. Además, estaba asociado a actitudes anómicas en el primer caso (indiferencia y búsqueda de soluciones personales ante los problemas sociales) y a la presencia de alienación política en el segundo caso (alta insatisfacción con todas las instituciones del sistema y con la democracia, rechazo generalizado a los partidos políticos, etc.). Todo esto ha llevado a que en los procesos electorales municipales se termine apoyando a candidatos que se asocian a la idea del "roba pero hace obra" (Meléndez, 2014) o que en la última elección para el Congreso el voto haya estado disperso en nueve agrupaciones donde el partido más votado obtuvo solo el 10.6\% de los votos válidos (Oficina Nacional de Procesos Electorales, 2020). Siendo lo anterior consistente con investigaciones que encuentran que la percepción deteriorada y la desconfianza difusa hacia el sistema político y jurídico es un factor de riesgo en la tolerancia ciudadana a la corrupción (Espinosa et al., 2020; Janos et al., 2018; Gächter \& Schulz, 2016).

En base a lo expuesto, el objetivo general del presente estudio fue examinar cómo actúan descripciones de procesos de control de la corrupción en el caso peruano, utilizando una muestra representativa a nivel nacional con una metodología experimental split-half. Al respecto, los diseños split-halfson frecuentemente utilizados en estudios por encuestas para determinar experimentalmente la diferencia entre dos variaciones del protocolo de levantamiento de información, donde siguiendo un criterio de aleatorización, una parte de la muestra fue expuesta a una descripción de eficacia y la otra a una descripción de ineficacia de los procesos anticorrupción (véase Ziniel, 2008). En este escenario, se hipotetiza que destacar o informar de una alta eficacia (versus una baja eficacia) en el control de la corrupción, reforzaría el clima emocional positivo y la identidad nacional peruana, siendo este efecto moderado por el interés en la política y la confianza previa en las instituciones vinculadas al ejercicio de la justicia procedimental -el efecto sería mayor en personas más interesadas y que confían en las instituciones. 


\section{Método}

\section{Muestreo y Participantes}

Para el proceso de selección de participantes se llevó a cabo un muestreo aleatorio estratificado polietápico a nivel nacional. El diseńo de la muestra es estratificado por regiones (Lima y Callao, Norte, Centro, Sur y Oriente), y al interior de las regiones existe una sub-estratificación por ámbito (urbano y rural). Al interior de estos ámbitos, se selecciona de manera aleatoria una muestra de ciudades y distritos, al interior de los mismos se seleccionan aleatoriamente manzanas, y se repite el procedimiento para la elección de viviendas. Finalmente, para la selección de personas se utilizan cuotas por sexo (hombres y mujeres) y por rango de edad (personas mayores de 18 años). La población estimada como universo muestral es de 23375001 personas.

Sobre este universo, la muestra definida con un $95 \%$ de confianza, asumiendo un $50-50 \%$ de heterogeneidad y un margen de error $\pm 2.8 \%$ a nivel nacional fue de 1254 participantes. Las edades de los participantes oscilaban entre los 18 y los 90 años $(M=38.92, D E=14.56)$. La distribución por sexo fue de $50.2 \%$ mujeres versus $49.8 \%$ hombres. Con relación al ámbito, el $87.96 \%$ de los participantes eran de zonas urbanas y el $12.04 \%$ de zonas rurales. En cuanto a las regiones Lima y Callao comprendía el $45 \%$ de la muestra, el Norte 19\%, el Centro $7 \%$, el Sur $18 \%$ y el Oriente el 11\%. Finalmente, la muestra de personas fue recopilada de 17 departamentos, 26 provincias y 88 distritos, lo que otorga un nivel de representatividad del $60 \%$ a nivel nacional. Del total de participantes 678 (54\%) fueron expuestos a la condición de alta eficacia del control de la corrupción, mientras que 576 (46\%) fueron expuestos a la condición de baja eficacia. Los grupos experimentales no presentan diferencias estadísticas en su distribución por sexo, ámbito y región. Con relación a la edad, un análisis de varianza muestra una diferencia significativa entre el grupo de alta eficacia, $M=38.07, D E=14.08$; y el grupo de baja eficacia $M=39.91, D E=15.06$, $F(1,1253)=5.017, p<.05, \eta^{2}=.04$, aunque con un tamańo del efecto pequeño. De lo anterior se concluye que los grupos son comparables 
en base a sus características socio-demográficas. Adicionalmente, los análisis controlando la edad y el sexo no modificaron los resultados por lo solo se reportan los resultados generales del estudio.

\section{Procedimiento y Medición}

El levantamiento de información del presente estudio fue ejecutado por el Instituto de Estudios Peruanos entre el 21 y 24 de septiembre del 2019, en un contexto de saliencia de escándalos políticos y corrupción en el país. La primera medida utilizada en el levantamiento de información fue la de Confianza en el Sistema Judicial, la cual fue evaluada con la siguiente pregunta: “¿Usted aprueba o desaprueba el desempeño del Poder Judicial?”. La escala de respuesta era dicotómica donde 1 =Aprueba y $2=$ Desaprueba. Una tercera opción de respuesta incluía los casos de personas que no sabían o no respondían a la pregunta. Estos casos fueron tratados como valores perdidos.

Posteriormente, los participantes del estudio, estuvieron expuestos de manera aleatoria bajo un criterio experimental split-half de dos grupos, a una de dos narrativas sobre corrupción que tenían una estructura común donde se presentaba un supuesto estudio actual que había identificado 856 casos de corrupción durante el 2012 (introduciéndose algunos ejemplos para darle verosimilitud a la narrativa). La narrativa aludía adicionalmente a la eficacia del sistema de justicia en el tratamiento de los casos de corrupción, estableciéndose una manipulación donde en la condición de alta eficiencia el 95\% de los casos eran sancionados, mientras para la condición de baja eficiencia solo el 5\% de los casos eran resueltos satisfactoriamente. En la tabla 2 se presentan los textos correspondientes a cada condición experimental.

Después de expuestos a alguna de las condiciones descritas, los participantes respondieron inicialmente a una pregunta de control de la manipulación, que permite asegurar la correcta comprensión del texto al que los participantes estuvieron expuestos. La pregunta en cuestión fue la siguiente: "según lo planteado en el texto anterior, ¿̇recuerda cuántos casos de corrupción han llegado a juicio?” y las respuestas a la pregunta 
fueron categorizadas en 12 alternativas tal como se describen a continuación $1=0 \%, 2=5 \%, 3=15 \%, 4=25 \%, 5=35 \%, 6=45 \%, 7=55 \%$, $8=65 \%$, 9=75\%, 10=85\%, 11=95\%, y $12=100 \%$. El tratamiento de los datos correspondientes a la medida de chequeo de la manipulación será reportado en el acápite de resultados.

\section{Tabla 2}

Estimulos sobre los procesos de corrupción y la eficacia en su resolución

\begin{tabular}{|c|c|}
\hline cia & cia \\
\hline $\begin{array}{l}\text { "Con respecto al tema de la } \\
\text { corrupción, en los últimos meses } \\
\text { han salido a la luz pública una serie } \\
\text { de casos de corrupción, pagos de } \\
\text { sobornos de grandes empresas a } \\
\text { políticos para financiar sus campañas, } \\
\text { obtención de beneficios para } \\
\text { conseguir contratos con el estado, } \\
\text { altísimas ganancias por especulación } \\
\text { inmobiliaria, entre otras situaciones. } \\
\text { En un estudio realizado en Perú se ha } \\
\text { establecido que de los } 856 \text { casos de } \\
\text { corrupción que han sido informados } \\
\text { e investigados hasta ahora, un } 95 \% \\
\text { ha llegado a juicio. En estos casos } \\
\text { se aplicaron duras sanciones a los } \\
\text { implicados". }\end{array}$ & $\begin{array}{l}\text { "Con respecto al tema de la } \\
\text { corrupción, en los últimos meses } \\
\text { han salido a la luz pública una serie } \\
\text { de casos de corrupción, pagos de } \\
\text { sobornos de grandes empresas a } \\
\text { políticos para financiar sus campañas, } \\
\text { obtención de beneficios para } \\
\text { conseguir contratos con el Estado, } \\
\text { altísimas ganancias por especulación } \\
\text { inmobiliaria, entre otras situaciones. } \\
\text { En un estudio realizado en Perú se ha } \\
\text { establecido que de los } 856 \text { casos de } \\
\text { corrupción que han sido informados } \\
\text { e investigados hasta ahora, solo un } \\
5 \% \text { ha llegado a juicio, dado que } \\
\text { en la gran mayoría ya pasó mucho } \\
\text { tiempo y han prescrito". }\end{array}$ \\
\hline
\end{tabular}

Luego de la respuesta al chequeo de la manipulación, los participantes fueron expuestos a un conjunto de escalas sobre Clima Socioemocional (positivo y negativo), Identidad Nacional Peruana, Eficacia Percibida del Control Social ante la corrupción e Interés en la Política.

Para la medición del Clima Socioemocional, se utilizó una adaptación de la escala desarrollada por Páez, Ruiz, Gailly, Kornblit, Wiesenfeld y Vidal (1997). La escala se presentó con el siguiente enunciado "pensando en la situación actual del Perú, ¿usted diría que el 
ambiente o clima social es...?” y se presentan los siguientes ítems: (1) de esperanza, esperanzado, (2) solidario, de ayuda mutua, (3) de confianza en las instituciones, y (4) de enojo, hostilidad, agresividad entre la gente". Cada ítem era respondido en una escala del 1 al 5, donde 1 era igual a nada y 5 era igual a mucho. Una sexta opción de respuesta comprendía el valor 6 , que correspondía a las personas que no sabían o no respondían. Esta última opción de respuesta fue tratada como caso perdido cuando aparecía. Los tres primeros ítems de la escala en conjunto configuran el indicador de clima emocional positivo que tuvo una confiabilidad aceptable de $\alpha=.67$. El ítem 4, se trabajó como un indicador de clima emocional negativo. Los resultados de esta escala fueron adicionalmente comparados con los resultados levantados en una muestra representativa de Lima en el año 2012 (Espinosa, Páez, Velázquez et al., 2016).

La medición de la Identidad Nacional Peruana se realizó con dos ítems extraídos de la escala de Identidad Nacional de Pinto et al., (2016). La escala se presentó con el siguiente enunciado: "A continuación, le presentamos una serie afirmaciones relacionadas con el ser peruano. Tomando en cuenta cómo reacciona el Perú ante la corrupción, ¿en qué medida está de acuerdo o en desacuerdo con estas frases?” y se presentan los siguientes ítems (1) Me siento orgulloso de ser peruano, y (2) Mi destino y mi futuro están ligados con el de los peruanos. Las respuestas a estas preguntas se registraban en una escala del 1 al 5, donde $1=$ Totalmente en desacuerdo y $5=$ Totalmente de acuerdo. Adicionalmente había una sexta categoría de respuesta que comprendía aquellos casos que no sabían o no respondían a la pregunta, y cuando fue utilizada fue tratada como un caso perdido. La consistencia interna de estas medidas fue aceptable, $\alpha=.52$, (cf. Mezulis, Abramson, Hyde, \& Hankin, 2004).

Para la evaluación de la Eficacia Percibida del Control Social de la Corrupción se desarrollaron dos ítems ad hoc que fueron antecedidos por el siguiente enunciado: "Indique, por favor, en qué medida está de acuerdo o en desacuerdo con cada una de las siguientes afirmaciones". Los ítems fueron los siguientes: (1) Nuestro sistema de justicia es eficaz 
para prevenir futuros casos de corrupción, y (2) Creo que el sistema judicial peruano es eficaz ante la corrupción. La respuesta a estos enunciados se realizaba en una escala del 1 al 5, donde $1=$ Totalmente en desacuerdo, y 5=Totalmente de acuerdo. Una sexta opción de respuesta registraba los casos que no sabían o no respondían a los enunciados, siendo tratada como valores perdidos. El indicador correspondiente se construyó promediando las respuestas a los dos ítems. La consistencia interna de la escala fue aceptable, $\alpha=.69$.

El Interés en la Política se evaluó con la siguiente pregunta: “¿Cuánto interés tiene usted en la política?” donde la escala de respuesta iba del 1 al 4, siendo $1=$ Nada y 4=Mucho. Una quinta opción registraba las respuestas de quienes no sabían o no tenían una respuesta definida, siendo estos casos tratados como perdidos. En este indicador las respuestas válidas se dicotomizaron uniendo los valores 1 y 2 como bajo interés en la política, y los valores 3 y 4 como alto interés en la política. Cabe resaltar que, si bien esta escala fue aplicada después de la manipulación experimental, al no apreciarse diferencias por grupos en este indicador, la escala será utilizada como variable moderadora de los efectos de la manipulación sobre los indicadores de Clima Socioemocional e Identidad Nacional.

\section{Resultados}

\section{Chequeo de la manipulación experimental}

Las 12 categorías de respuesta al chequeo de la manipulación dan cuenta que las respuestas al porcentaje de casos de corrupción en los participantes expuestos a la condición de baja eficacia del control de los procesos de corrupción, tuvo una media de $3.4(D E=2.6)$, es decir estimaciones que van desde el $0 \%$ hasta el $45 \%$ de casos juzgados, cuando la información provista daba cuenta de un 5\%; mientras los participantes expuestos a la condición de alta eficacia en el control de los procesos de corrupción presentaban una media de 8.1 ( $D E=3.6)$, es decir estimaciones que van desde el $35 \%$ hasta el $100 \%$ de efectividad 
en el control de la corrupción, cuando la información provista daba cuenta de un $95 \%$ de eficacia. Las diferencias entre ambas condiciones es estadísticamente significativa, $F(1,1254)=710.822, p<.001, \eta^{2}=.36$.

Sobre el control de la manipulación se toma la decisión de eliminar un $15 \%$ de los participantes, debido a que sus respuestas se alejaban sustancialmente de la información dada, lo que hacía pensar que no habían prestado atención o no habían entendido el texto presentado correctamente. Así, aquellas personas que respondían más de un 15\% de eficacia en las acciones anticorrupción, en la condición de baja eficacia; y aquellas que respondían menos de un 75\% de eficacia en las acciones anticorrupción en la condición de alta eficacia no fueron consideradas en los análisis subsiguientes.

Impacto de la información sobre la eficacia del control de la corrupción en la eficacia percibida del control social, el clima socioemocional y la identidad nacional

Para contrastar el impacto de la información sobre la eficacia del control de la corrupción, se procesó una correlación punto-biserial entre la manipulación ( 1 =baja eficacia, 2 = alta eficacia) y las variables dependientes: clima socioemocional positivo, clima socioemocional negativo, identidad nacional peruana y la eficacia percibida del control social de la corrupción.

Tras la eliminación de los participantes que presentaron problemas en la recordación de la información correspondiente a la condición experimental a la que fueron expuestos, los resultados muestran que la información sobre eficacia en los procesos de atención a los casos de corrupción se relaciona directamente con la percepción de eficacia del control social de la corrupción, $r_{p b}(929)=.07, p<.05$, y mejora la balanza del clima socioemocional, consistente en un indicador obtenido del puntaje de clima socioemocional positivo menos el puntaje del clima socioemocional negativo, $r_{p b}(964)=.07, p<.05$. Asimismo, aumenta tendencialmente el clima socioemocional positivo, $r_{p b}(964)=.043$, $p=1$, en particular aumenta la percepción de un clima positivo de solidaridad, 
$r_{\mathrm{pb}}(964)=.07, p<.05$, y disminuye tendencialmente el clima negativo de enojo, $r_{p b}(964)=-.05, p=.07$. No se encontró un efecto de la información sobre la identidad nacional peruana (ver las medias de las variables dependientes por condición en la tabla 3).

\section{Tabla 3}

Medias y desviaciones estándar de las variables dependientes por condición (baja versus alta eficacia)

\begin{tabular}{lcccc}
\hline & \multicolumn{4}{c}{ Condición Experimental } \\
\cline { 2 - 5 } & \multicolumn{2}{c}{ Baja eficacia } & \multicolumn{2}{c}{ Alta eficacia } \\
\cline { 2 - 5 } & $\mathrm{M}$ & $\mathrm{DE}$ & $\mathrm{M}$ & $\mathrm{DE}$ \\
\hline Eficacia Percibida de Control Social & 2.24 & .83 & 2.35 & .84 \\
Clima Socioemocional Positivo & 2.20 & .70 & 2.27 & .73 \\
Clima Socioemocional Negativo & 3 & 1.11 & 2.9 & 1 \\
Balanza de Clima Socioemocional & -.80 & 1.26 & -.62 & 1.19 \\
Identidad Nacional Peruana & 3.57 & .81 & 3.50 & .87 \\
\hline
\end{tabular}

Efectos de interacción de la condición experimental con las variables moderadoras de interés en la política y confianza en el sistema judicial (poder judicial)

Un análisis de varianza usando como variables independientes la condición experimental (baja versus alta eficacia) y el interés en la política dicotomizado (bajo versus alto interés) y como variable dependiente la balanza de clima socioemocional, encontró una interacción significativa $F(1,1021)=4.027, p<.05$. Así, en los participantes interesados en la política, la información de baja eficacia empeora la balanza de clima socioemocional, mientras que la información de alta eficacia la mejora. Por otra parte, en los participantes con bajo interés en la política, la balanza de clima socioemocional es igual en ambas condiciones experimentales, para ver las medias de la balanza del clima socioemocional por condición e interés en la política véase el gráfico 1. 


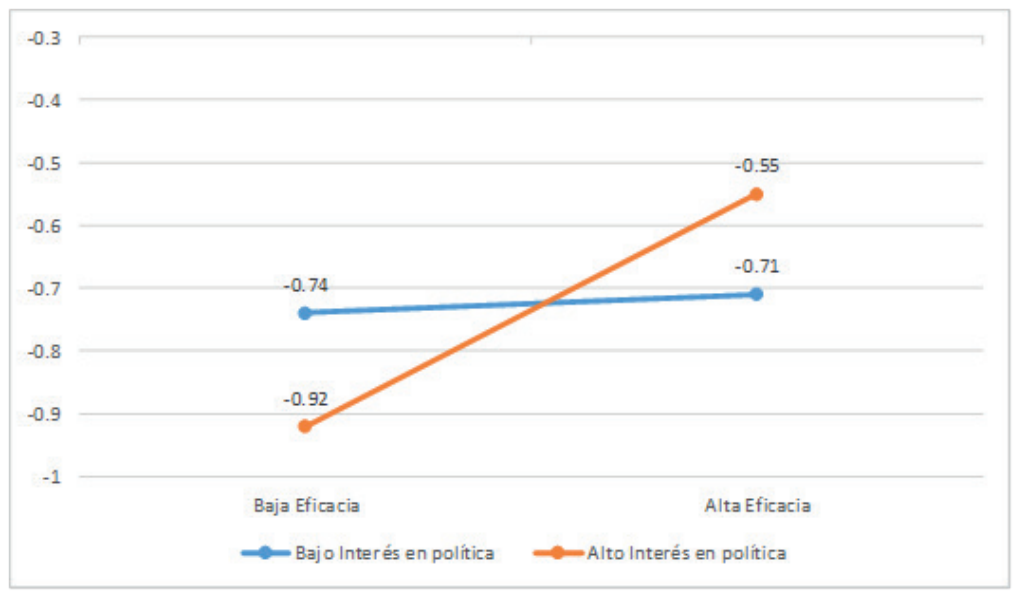

Gráfico 1. Efecto de Interacción entre la Condición Experimental (Baja Eficacia versus Alta Eficacia) y el Interés en la Política (Bajo Interés versus Alto Interés) en la Balanza de Clima Socioemocional.

El análisis de varianza usando como variables independientes la condición experimental (baja versus alta eficacia) y el interés en la política dicotomizado (bajo versus alto interés) y como variable dependiente la medida de identidad nacional no muestra interacciones significativas.

Con relación a la confianza en el sistema judicial (poder judicial), cuando a los participantes que aprueban al mismo de forma disposicional (un 16\% del total de la muestra), se le dice que la eficacia en el control de la corrupción es alta, aumenta su identificación con el Perú, es decir, se encontró un efecto de interacción, $F(1,972)=3.767, p<.05$. Donde los participantes que aprueban el sistema judicial en la condición de alta eficacia del control de la corrupción puntúan más alto en identidad nacional que el grupo que aprueba el sistema judicial expuesto a la condición de baja eficacia. Por otra parte, el grupo que desaprueba el poder judicial expuesto a la información de alta eficacia del control de la corrupción puntúa más bajo en identidad nacional, que el grupo en la condición experimental de baja eficacia (ver gráfico 2 ). 


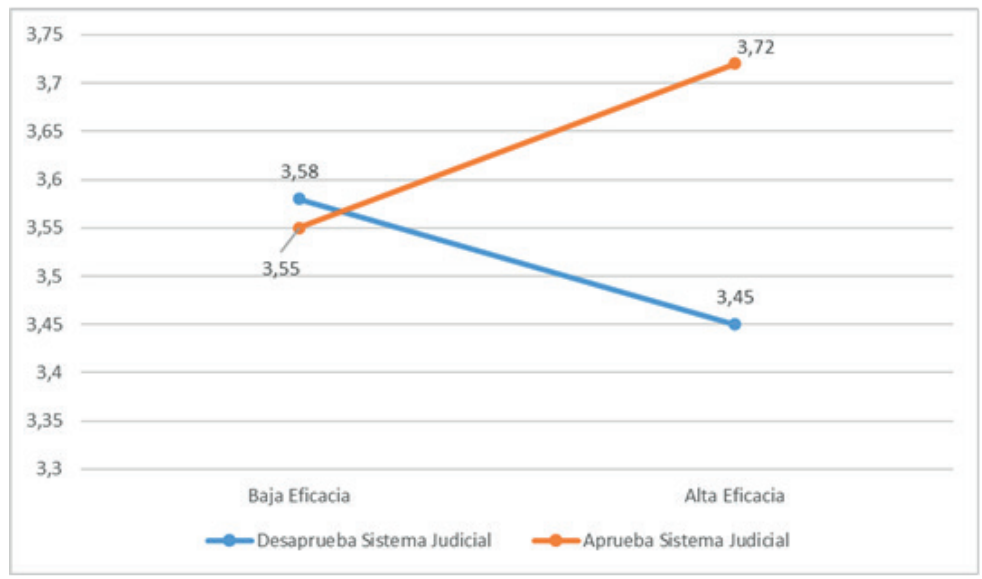

Gráfico 2. Efecto de Interacción entre la Condición Experimental (Baja Eficacia versus Alta Eficacia) y la Aprobación del Sistema Judicial (Desaprueba versus Aprueba) en la Identidad Nacional.

Un análisis de varianza donde se usan como variables independientes la condición experimental (baja versus alta eficacia) y la confianza en el poder judicial (baja versus alta aprobación), y como variable dependiente la balanza de clima socioemocional, no muestra interacciones significativas entre las variables independientes.

\section{Evolución del Clima Socioemocional en el tiempo: comparación con una medición del 2012}

Finalmente, se compararon las medias de clima socioemocional positivo y negativo de la región Lima y Callao del presente estudio, con los indicadores de clima socioemocional obtenidos el 2012 en una muestra representativa de las mismas ciudades (ver Espinosa et al. 2016). Con relación al clima socioemocional positivo, este es mayor en la muestra del presente estudio $(M=2.30)$, que en la muestra del 2012 $(M=2.14), t=7.29, p<.001$. Además, el clima socioemocional positivo se intensifica con mayor intensidad en el grupo expuesto a la condición 
de alta eficacia de control de la corrupción (ver gráfico 3), mientras que en la condición de baja eficacia del control de la corrupción se incrementa el clima socioemocional negativo (ver gráfico 3).

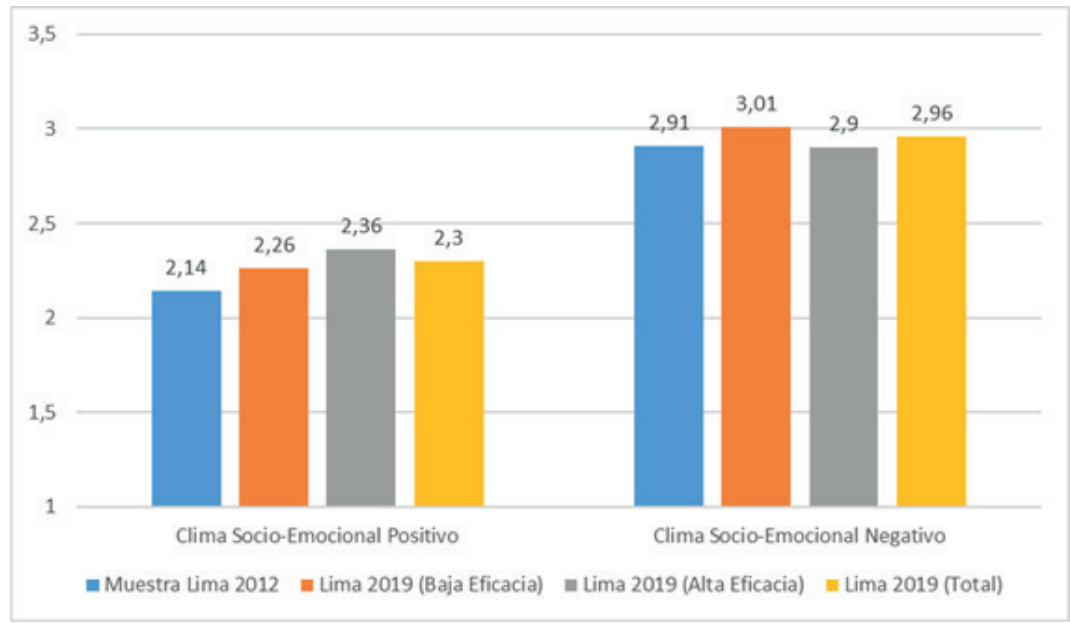

Gráfico 3. Evolución del Clima Socioemocional Positivo y Negativo en el tiempo

\section{Discusión y Conclusiones}

El estudio confirma parcialmente que destacar el monitoreo o control eficaz de la corrupción no solo disminuye la prevalencia de esta directamente, sino que tiene efectos positivos en la cohesión social. Un discurso positivo en relación con el control social, primero, aumenta la percepción de eficacia con que este control se aplica en general, confirmando que la información tiene un impacto, aunque de efecto pequeño $(r=.07)$.

En segundo lugar, una información de alta eficacia en el control de la corrupción mejora el clima socioemocional como se esperaba. El efecto en este caso también es pequeño $(r=.07)$ y sobre todo se produce en una minoría interesada en política (aproximada un 38\% de 
los participantes). El discurso de control social eficaz aumenta el clima socioemocional positivo y disminuye el negativo. Sin embargo, dado que este último predomina, lo que se logra es que la percepción del clima sea menos negativa cuando el discurso enfatiza los éxitos de control social. En este estudio como en otros, predomina una visión crítica del clima emocional ya que se constata que hay mayor percepción de ira colectiva que de emociones positivas (véase Páez, Espinosa \& Bobowik, 2013). Esta situación es frecuente en América Latina, aunque no en Europa, probablemente debido a la prevalencia de mayores conflictos y problemas sociales en las naciones de la primera región. (Páez et al., 2013). Las comparaciones longitudinales con relación al clima socioemocional en el pasado reciente refuerzan estas conclusiones. Cuando los mecanismos de control social del grupo se perciben como eficaces, las personas perciben más emociones colectivas positivas como la solidaridad, como mostraron las comparaciones entre las medias de la muestra representativa del 2012 y la muestra del presente estudio. Por el contrario, cuando los mecanismos de control social de la corrupción se perciben como ineficaces o inexistentes, las personas perciben ira colectiva, como mostraron las comparaciones entre la muestra de Lima actual informada de baja eficacia, que informaba de más ira colectiva que la muestra de Lima en el 2012, siendo esto es congruente con los hallazgos del estudio de Pinto et al., (2016).

El discurso de alta eficacia en el control de la corrupción no tuvo efecto directo sobre la identidad nacional, como ocurrió en un estudio realizado en Portugal, (Pinto et al., 2016), aunque en el estudio realizado en Chile por Hatibovic et al., (2020) se aprecia un comportamiento de similar al de la muestra peruana del presente estudio. Probablemente esto tiene relación con la crisis social y estatal que afecta en particular a las naciones latino-americanas. También se puede explicar por la baja confianza en las instituciones en ambos países. Para que un ritual o acción colectiva de control y reparación social sea efectivo es necesario que se perciba un contexto institucional positivo o al que se haga confianza (véase Mathias et al, 2020 cuyo meta-análisis encontró que en los países en los que había más confianza en el gobierno y un mayor 
apoyo a democracia, la influencia de un ritual colectivo en la percepción positiva de la sociedad era mayor, ya que la asociación entre la percepción de la eficacia de las Comisiones de la Verdad y el clima emocional era más fuerte). Coherentemente con lo antes argumentado, se dio un efecto positivo de la información de control eficaz sobre la identidad en interacción con la visión previa de la justicia. La gente que previamente tiene una alta confianza en el poder judicial, cuando se le dice que la eficacia del control es alta, aumenta la identificación. Esto sugiere que, si existe en los individuos una evaluación favorable del contexto institucional, la información positiva que reciban sobre la eficacia en el control de la corrupción tenderá a tener un efecto positivo en la identificación nacional.

\section{Referencias}

Agerberg, M. (2019). The Curse of Knowledge? Education, Corruption, and Politics. Political Behavior, 41: 369. https://doi. org/10.1007/s11109-018-9455-7

Anduiza, E., Gallego, A., \& Muñoz, J. (2013). Turning a blind eye: Experimental evidence of partisan bias in attitudes toward corruption. Comparative Political Studies, 46(12), 1664-1692.

Belle, N. \& Cantarelli, P. (2017). What Causes Unethical Behavior? A Meta-Analysis to Set an Agenda for Public Administration Research. Public Administration Review, 77 (3), 327-339 doi: 10.1111/puar.12714.

Bozerman, M.H. \& Gino, F. (2012). Behavioral Ethics: Toward a Deeper Understanding of Moral Judgment and Dishonesty. Annual Review of Law and Social Science, 8:85-104.

Caillier, J. (2010). Citizen trust, political corruption, and voting behavior: Connecting the dots. Politics and Policy, 38(5), 1015-1035.

Chaparro, H. (2018). Afectos y desafectos. Las diversas subculturas politicas en Lima. Lima, Perú: Instituto de Estudios Peruanos. 
Charron, N., \& Rothstein, B. (2016). Does education lead to higher generalized trust? The importance of quality of government. International Journal of Educational Development, 50, 59-73.

Durkheim, E. (1895/1982). The Rules of Sociological Method. New York: The Free Press.

Espinosa, A., Pacheco, M., Acosta, Y., et al., (2020). Ideology and Political Cynicism: Effects of Authoritarianism and Social Dominance on Perceptions about the Political System in 11 Ibero-American Countries. Manuscrito en revisión.

Espinosa, A., Páez, D., Velázquez, T., Cueto, R. M., Seminario, E., Sandoval, S., Reátegui, F., \& Jave, I. (2017). Between remembering and forgetting the years of political violence: Psychosocial impact of the Truth and Reconciliation Commission in Peru. Political Psychology, 38, 849-866. doi:10.1111/pops.12364

Gächter, S., \& Schulz, J. (2016). Intrinsic honesty and the prevalence of rule violations across societies. Nature, 531, 496-499. doi:10.1038/nature17160

Hatibovic, F., Pinto, I. \& Páez, D. (2020). Corruption, perceived social control and national identification in Chilean students. Manuscrito en revisión.

Heath, A.F., Richards, L. \& de Graaf, N.D. (2016) Explaining Corruption in the Developed World: The Potential of Sociological Approaches. Annual Review of Sociology, 42, 51-79.

Hogg, M. \& Vaughan, G. (2013). Social Psychology. London, UK: Pearson.

Instituto de Estudios Peruanos (2018) Los peruanos y la corrupción. Recuperado de https://iep.org.pe/wp-content/ uploads/2018/12/Informe-OP-Diciembre-2018-Los-peruanosy-la-corrupci\%C3\%B3n-2.pdf

Instituto de Estudios Peruanos (2019a). IEP Informe de Opinión - Diciembre 2019. Evaluación de autoridades e instituciones. Recuperado de https://iep.org.pe/wp-content/uploads/2019/12/ Informe-OP-Dic-2019-eval-autoridades-e-instituciones.pdf 
Instituto de Estudios Peruanos (2019 b). IEP Informe de Opinión Mayo 2019. Congreso, Poder Judicial y Fiscalía. Recuperado de https://iep.org.pe/wp-content/uploads/2019/05/Informe-OPMayo-2019-Congreso-PJ-y-Fiscal\%C3\%ADa.pdf

Janos, E., Espinosa, A., \& Pacheco, M. (2018). Bases Ideológicas de la Percepción del Sistema Normativo y el Cinismo Político en Adultos de Sectores Urbanos del Perú. Psykhe, 27(1), 1-14. doi: 10.7764/psykhe.27.1.1176

Jaramillo, N. J. (2011). ¿Existe alguna relación entre la corrupción política y la democracia? Breve reflexión teórica dirigida a establecer sus posibles articulaciones. OPERA - Observatorio de Politicas, Ejecución y Resultados de la Administración Pública, (11), 241-257.

Judge, W.Q., McNatt, D.B. \& Xu, W. (2011). The antecedents and effects of national corruption: A meta-analysis. Journal of World Business, 46(1), 93-103.

Marques, J. M., Páez, D., \& Abrams, D. (1998). Social identity and intragroup differentiation as subjective social control. En $S$. Worchel, J. F. Morales, D. Páez, \& J.-C. Deschamps (Eds.), Social identity: International perspectives (pp. 124-141). London, England: Sage.

Mathias, A., Páez, D., Espinosa, A., Sandoval, S., Alzugaray, C., Arnoso, M. Cárdenas, M., da Costa, S., Reyes, C., Rimé, B. \& Zubieta, E. (2020). The association between Truth Commissions evaluation, emotional climate and institutional trust: comparison and meta-analysis of surveys in six South American. International Journal of Social Psychology, 35(2), 203-245, doi:10.1080/02134748.2020.1721053

Mezulis, A. H., Abramson, L. Y., Hyde, J. S., \& Hankin, B. L. (2004). Is There a Universal Positivity Bias in Attributions? A MetaAnalytic Review of Individual, Developmental, and Cultural Differences in the Self-Serving Attributional Bias. Psychological Bulletin, 130(5), 711-747. doi:10.1037/0033-2909.130.5.711 
Páez, D., Ruiz, J. I., Gailly, O., Kornblit, A. L., Wiesenfeld, E., \& Vidal, C. M. (1997). Clima emocional: Su concepto y medición mediante una investigación transcultural. Revista de Psicología Social, 12(1), 79-98.

Páez, D., Espinosa, A. \& Bobowik, M. (2013). Emotional climate: How is it shaped, fostered, and changed? En D. Hermans, B. Rimé \& B. Mesquita (Eds.), Changing Emotions (pp. 113-119). London, England: Psychology Press.

Philip, M. \& David-Barrett, E. (2015). Realism About Political Corruption. Annual. Review of Political Science, 18,387-402

Pinto, I. R., Marques, J. M., \& Páez, D. (2016). National identification as a function of perceived social control: A subjective group dynamics analysis. Group Processes \& Intergroup Relations, 19(2), 236-256. doi:10.1177/1368430215577225.

Proética (2019) XI Encuesta Nacional Anual sobre Percepciones de Corrupción. Recuperado de https:/www.proetica.org.pe/ contenido/xi-encuesta-nacional-sobre-percepciones-de-lacorrupcion-en-el-peru-2019/

Quiroz, A. (2013). Historia de la Corrupción en el Perú. Lima, Perú: Instituto de Estudios Peruanos.

Rose-Ackerman, S. (2001), La corrupción y los gobiernos: Causas, consecuencias y reforma. Madrid, Espana: Siglo XXI.r

Rosenthal, R. (2005). Binomial Effect Size Display. Encyclopedia of Statistics in Behavioral Science, 1, 157-158. https://doi. org/10.1002/0470013192.bsa050

Rosenthal, R., \& Rubin, D.B. (1982). A simple general purpose display of magnitude of experimental effect. Journal of Educational Psychology, 74, 166-169.

Transparency International, (2018). Corruption Perception Index. Recuperado de: http://www.transparency.org/cpi2018.

Pring, C. \& Vrushi, J. (2019.) Global Corruption Barometer Latin American o the Caribbean 2019. Citizens' views and experiences of corruption. Berlin, Germany: International Transparency. Recuperado de https://www.transparency.org/es/publications/ 
global-corruption-barometer-latin-america-and-thecaribbean-2019.

UK Department for International Development (2015) Why corruption matters: understanding causes, effects and how to address them. UK: International Department for International Development. Ziniel, S. (2008). Split-half. In P.J. Lavrakas (Ed.). Encyclopedia of Survey Research Methods (vol. 2, pp.833-834). SAGE. http:// dx.doi.org/10.4135/9781412963947.

Recibido: 2020-08-03 Revisado: 2020-12-07 Aceptado: 2021-03-18 